\title{
On the analysis of time-periodic nonlinear dynamical systems
}

\section{S C SINHA}

Nonlinear Systems Research Laboratory, Department of Mechanical Engineering, Auburn University, AL 36849, USA

e-mail: ssinha@eng.auburn.edu

\begin{abstract}
In this study, a general technique for the analysis of time-period nonlinear dynamical systems is presented. The method is based on the fact that all quasilinear periodic systems can be replaced by similar systems whose linear parts are time invariant via the well-known Liapunov-Floquet (L-F) transformation. A general procedure for the computation of L-F transformation in terms of Chebyshev polynomials is outlined. Once the transformation has been applied, a periodic orbit in original coordinates has a fixed point representation in the transformed coordinates. The stability and bifurcation analysis of the transformed equations are studied by employing the time-dependent normal form theory and time-dependent centre manifold reduction. For the two examples considered, the three generic codimension-one bifurcations, viz, Hopf, flip and tangent, are analysed. The methodology is semi-analytic in nature and provides a quantitative measure of stability even under critical conditions. Unlike the perturbation or averaging techniques, this method is applicable even to those systems where the periodic term in the linear part does not contain a small parameter or a generating solution does not exist due to the absence of the time-invariant term in the linear part.
\end{abstract}

Keywords. Nonlinear dynamical systems; Liapunov-Floquet transformation; centre manifold reduction; normal form theory; bifurcations; time-invariant forms.

\section{Introduction}

The study of systems governed by a set of ordinary differential equations is of great importance in diverse branches of science and engineering. Numerous practical applications can be found in the areas of quantum mechanics, systems and controls and dynamic stability of structures under oscillating loads. In particular, such problems arise in the dynamics of rotating systems such as helicopter blades (Bramwell 1976; Johnson 1980) and rotor-bearing systems (Lalanne \& Ferraris 1990). Mathematical modelling of such systems results in 
nonlinear non-autonomous differential equations which contain explicit periodic functions of time. Periodic solutions of these equations physically represent steady-state operations under various conditions. The stability of these periodic solutions (or orbits) is determined by the equations of perturbed motion about the periodic solutions. In many situations, the linearized perturbed equations may be sufficient for the prediction of stability, and therefore the problem reduces to a set of linear ordinary differential equations with periodic coefficients. The same mathematical problem also arises in the study of nonlinear autonomous systems when the stability of a particular periodic solution needs to be investigated. Besides the stability issues, the linear control problems associated with rotating systems can also lead to the same type of equations. For example, the Individual-Blade-Control (IBC) technique, used in the control of a helicopter rotor-blade system, produces a set of equations with periodic coefficients (Kretz 1976; Mckillip 1985). Therefore the analysis of this special class of time-varying systems has been deemed extremely important.

Hill's method (Lindh \& Likins 1970; Yakubovitch \& Starzhinskii 1975), perturbation techniques (Stoker 1950; Nayfeh 1973) and Floquet's theory (Coddington \& Levinson 1955; Floquet 1983) are some of the most commonly used mathematical methods in the analysis of such systems. It is well known that Hill's approach is not suitable for digital implementation, especially if one has to deal with a large-scale system. The perturbation methods have their own limitations due to the fact that they can only be applied to systems where the periodic coefficients can be expressed in terms of a small parameter. Therefore, Floquet analysis coupled with a numerical integration code has served as the main tool in various applications (Peters \& Hohenemser 1971; Friedmann et al 1977; Gaonkar et al 1981; Sinha \& Wu 1991; Wu \& Sinha 1994). Floquet analysis is a powerful technique which can be easily implemented on a computer and holds most promise in the analysis of large-scale periodic systems. According to this technique, stability analysis requires the eigen-analysis of the 'Floquet Transition Matrix' (FTM) which is simply defined as the state transition matrix at the end of one period. Eigen-analysis and the evaluation of FTM are the two major computational problems encountered in the analysis of linear periodic systems of large dimensions. Since the FTM is nonsymmetric, the eigen-analysis problem is a difficult one. Some progress has been made in this direction (Cullum \& Willoughby 1986; Gaonkar \& Peters 1986), however, the computation of FTM for large-scale systems is still a challenging task. Most commonly a fourth or higher-order Runge-Kutta type numerical code has been used in a 'single pass' scheme for an efficient computation of the FTM (Friedmann et al 1977; Gaonkar et al 1981). Very recently, Sinha and associates (Sinha \& Wu 1991; Joseph et al 1993; Sinha et al 1993; Wu \& Sinha 1994) have developed a new technique for the analysis of large-scale periodic systems. It has been shown that the proposed technique is numerically several times faster than the standard codes and at the same time it can also be applied in the symbolic form (Sinha \& Juneja 1991).

Although the linearized equations play an important role in the stability analyses, they fail to provide answers to many questions associated with the nonlinear periodic systems. Questions such as how does the solution depend on a control parameter? What kind of motion takes place after the loss of stability of the periodic state? Is it possible to identify the types of stability loss and critical values of the control parameters in each case etc? In order to answer such questions one must investigate the nonlinear equations of perturbed motion 
including a bifurcation analysis of the periodic orbit. In many instances certain methods of nonlinear dynamics can be applied to obtain information of significant value. It is known (Arnold 1988) that the simplest loss of stability of a periodic orbit constitutes a degenerate problem of codimension 1 . However, if a pair of complex multipliers simultaneously cross the unit circle of the complex plane, then we have the Hopf bifurcation of periodic orbits. The details are given by Guckenheimer \& Holmes (1983) and Arnold (1988). For the case of a codimension 2 bifurcating periodic orbit, some qualitative mathematical results have also be obtained (Chow \& Wang 1985). Qualitative analyses certainly provide good insight into the problems, but for engineering applications quantitative methods are indispensable.

Perturbation and averaging methods (Bogoliubov \& Mitropolsky 1961; Nayfeh 1973; Sanders \& Verhulst 1985) are suitable for relatively smaller systems and in general, their applications are limited to systems where the periodic terms as well as the nonlinearities can be expressed in terms of a suitable small parameter. Hopf bifurcation of Duffing's oscillator and nonlinear Mathieu's equations are discussed by Awreicewicz (1989) through an application of perturbation and harmonic balance methods. On the other hand, one can apply standard numerical techniques associated with boundary value problems to analyse the situation. These techniques are basically shooting methods and provide strategies for calculating branch points and new branches of bifurcating solutions. These methods have been exploited by several authors (Seydel 1981, 1987, 1988; Doedel \& Kernévez 1986). Shooting methods are quite reliable but they are certainly not free from numerical instability difficulties. At the same time, when a system is non-autonomous, the trajectories can cross themselves and it is difficult to obtain a general structure of the motion through a purely numerical scheme. An attractive alternate method of analysis is provided by the technique called 'point mapping'. The idea was introduced by Poincaré (1899) and later developed by Birkhoff (1966), Arnold (1988) and Bernussou (1977). In this approach the continuous-time periodic system is reformulated as discrete-time events by defining a point mapping called the Poincaré map. Thus the original non-autonomous differential system is replaced by a set of difference equations which do not explicitly depend on time. In principle these are easier to analyse and simulate on a digital computer. However, one faces serious computational difficulties in application of this technique to real engineering problems even if the dimensions are small. In order to obtain the corresponding difference equations, one must construct an exact or approximate solution of a system of nonlinear differential equations. Exact solutions are only possible in very special cases, such as those of impulsive excitation problems discussed by Hsu and his associates (Hsu \& Cheng 1973, 1974; Flashner \& Hsu 1983; Hsu 1987). Since one must settle for an approximate representation of the point mapping, recent studies (Lukes 1982; Flashner \& Hsu 1983; Guttalu \& Flashner 1989, 1990) have suggested the use of Runge-Kutta type algorithm and perturbation technique for obtaining a truncated version of the Poincare map. Following this approach one can discuss the bifurcation of periodic solutions to other possible periodic motions or to quasiperiodic and aperiodic solutions (Lindtner $e t$ al 1990).

One other viable approach is to use the Liapunov-Floquet theorem which allows transformation of the quasilinear periodic systems into a new set of similar equations whose linear parts are time-invariant. However, it is not a simple task to compute this transformation 
matrix for a general periodic system. For certain special class of linear systems, it is possible to obtain the Liapunov-Floquet transformations as indicated by Lukes (1982). In order to determine such a transformation for a general periodic system, one must compute the STM as an explicit function of time. Recently we (Pandiyan et al 1993; Sinha \& Joseph 1994; Sinha \& Pandiyan 1994; Pandiyan \& Sinha 1995) have been successful in developing a computational procedure through which the Liapunov-Floquet (L-F) transformation can be obtained in terms of Chebyshev polynomials which is suitable for algebraic manipulations. The inverse of the L-F transformation can also be computed by a similar procedure considering the adjoint system equation.

The development of a procedure for computing these transformation matrices has given a clear edge in dealing with a wide range of problems associated with periodically varying systems. In this paper, a quantitative analysis of nonlinear dynamical systems with periodic coefficients has been presented through an application of the Liapunov-Floquet (L-F) transformation. It is shown that the original quasilinear periodic system can be transformed to a dynamically similar form in which the linear part is time-invariant. The analysis of the transformed equations has been carried out through the use of time-dependent normal form theory. The solutions thus obtained are mapped back to the original coordinates by applying the inverse L-F transformations and compared with the numerical results obtained by a Runge-Kutta type algorithm. The method is also applicable to systems undergoing bifurcations. Such problems are referred to as 'critical cases' and have been studied through an application of the centre manifold theory (Malkin 1962; Carr 1981). For brevity, only codimension 1 bifurcations are considered. In order to demonstrate the effectiveness of the proposed analysis procedure, two examples have been studied in detail. The first example consists of a nonlinear Mathieu equation, the L-F transformation of which has been computed using the Chebyshev polynomials as described in $\S 3$. The solutions of this example have been obtained in stable and centre manifolds for some typical sets of system parameters. It has been shown that the proposed technique is applicable to a wide class of problems including the situations where the generating solutions do not exist and/or the parameter multiplying the linear periodic terms are no longer small. It is also shown that in many cases it is possible to obtain approximate analytical solutions which compare extremely well with the numerical solutions. The results obtained by the traditional averaging method are also presented for comparison purposes.

As a second example, the bifurcation problem of a double inverted pendulum subjected to periodic loading is selected. The Hopf bifurcation in a double inverted pendulum subjected to a tangential static load has been studied by Sethna \& Shapiro (1977) and thereafter many researchers have contributed on various bifurcation aspects of such an autonomous system. However, when the double pendulum is subjected to a periodic load, the system becomes non-autonomous. Periodic bifurcations of such a pendulum has been reported by Flashner \& Hsu (1983) by the method of point mappings. In this paper, the dynamics of this four-dimensional system undergoing a single Hopf bifurcation or a single flip bifurcation is investigated in a two-dimensional centre manifold or a single-dimensional centre manifold, respectively by applying the time-dependent normal form theory and centre manifold reduction. The results of such analyses are verified by using numerical simulations. 


\section{Background}

\subsection{Mathematical structure of periodic systems}

In general, many problems of mechanical systems can be reduced to a set of nonlinear ordinary differential equations of the form,

$$
\dot{\mathbf{y}}=\mathbf{g}(y, \lambda, t),
$$

where $\mathbf{y}$ is an $n$ state vector, $g(\mathrm{)}$ is a continuous nonlinear function of $y$ and $t$ and $\lambda$ is a set of control parameters.

Let $\bar{y}(t)$ denote a known periodic solution (of period $T$ ) of (1) such that the perturbation $x(t)$ about the periodic motion $\bar{y}(t)$ can be defined as

$$
y(t)=\bar{y}(t)+x(t) .
$$

Substituting (2) in (1) and expanding the right-hand side in Taylor series about $\bar{y}(t)$ yields

$$
\dot{x}_{i}(t)=\left.\frac{\partial g_{i}}{\partial y_{j}}\right|_{\bar{y}(t)} x_{j}+\left.\frac{1}{2} \frac{\partial^{2} g_{i}}{\partial y_{j} \partial y_{k}}\right|_{\bar{y}(t)} x_{j} x_{k}+\left.\frac{1}{3 !} \frac{\partial^{3} g_{i}}{\partial y_{j} \partial y_{k} \partial y_{l}}\right|_{\bar{y}(t)} x_{j} x_{k} x_{l}+\cdots
$$

The above equation may be rewritten as

$$
\dot{\mathbf{x}}=\mathbf{A}(t, \lambda) x+\mathbf{f}_{2}(x, t, \lambda)+\mathbf{f}_{3}(x, t, \lambda)+\cdots+\mathbf{f}_{k}(x, t, \lambda)+\mathbf{O}\left(|x|^{k+1}, t\right)
$$

or

$$
\dot{\mathbf{x}}=\mathbf{A}(t, \lambda) x+\mathbf{F}(x, t, \lambda)
$$

where $\mathbf{f}_{k}\left(\mathrm{)}\right.$ contain homogeneous monomials in $x_{i}$ of order $k . \mathbf{A}(t, \lambda), \mathbf{f}_{k}(x, t, \lambda)$ and $\mathbf{F}(x, t, \lambda)$ (suitably defined in terms of $f_{k}()^{\prime}$ 's) are $T$ periodic functions. The linear part of (4) is, of course, given by

$$
\dot{\mathbf{x}}=\mathbf{A}(t, \lambda) x ; \quad \mathbf{A}(t, \lambda)=\mathbf{A}(t+T, \lambda) .
$$

The stability and response of (5) can be discussed using the well-known Floquet theory.

We are interested in the analysis of (4) and determine its behaviour as the control parameter $\lambda$ varies.

\subsection{Results from Floquet theory (Floquet 1983; Coddington \& Levinson 1955)}

Theorem 1. Each state transition matrix (STM), $\Phi$ of (5) can be written as the product of two $n \times n$-matrices as

$$
\Phi(t)=\mathbf{L}(t) e^{t C}
$$

where $\mathbf{L}(t)$ is $T$-periodic and $\mathbf{C}$ is a constant $n \times n$ matrix. $\mathbf{L}(t)$ and $\mathbf{C}$, in general, are complex. 
Remark 1. There exists a complex matrix $\mathbf{C}$ such that

$$
\mathbf{M}=e^{C T},
$$

where $\mathbf{M}$ can be expressed as

$$
\mathbf{M}=\Phi^{-1}(o) \Phi(T) .
$$

Remark 2. $\mu_{i}$, the eigenvalues of $\mathbf{M}$ are called the characteristic multipliers and the stability condition can be expressed as $\left|\mu_{i}\right|<1, i=1,2, \ldots, n$.

\section{COROLLARY 1}

Each state transition matrix $\Phi(t)$ can also be factored as

$$
\Phi(t)=\mathbf{Q}(t) e^{\mathbf{R} t}
$$

where the matrix $\mathbf{Q}(t)$ is real and periodic with period $2 T$ and $\mathbf{R}$ is an appropriate real matrix.

\section{COROLLARY 2}

The Liapunov-Floquet transformation

$$
\mathbf{x}(t)=\mathbf{L}(t) z(t)
$$

reduces the original time-varying system (5) to

$$
\dot{z}(t)=\mathbf{C} z(t)
$$

which is time-invariant.

Moreover, the 2T-periodic transformation

$$
x(t)=\mathbf{Q}(t) z(t)
$$

produces a real representation given by

$$
\dot{z}(t)=\mathbf{R} z(t)
$$

\section{Computation of L-F transformation matrix via Chebyshev polynomials}

It has been shown by Sinha and associates (Sinha \& Juneja 1991; Sinha \& Wu 1991; Joseph et al 1993; Sinha et al 1993; Wu \& Sinha 1994) that the STMs of linear periodic systems can be obtained in terms of the shifted Chebyshev polynomials of the first kind. The technique is efficient and since the STM is expressed as an explicit function of time $t$, it is suitable for algebraic manipulations as well. In fact, if the dimension is small, the STM can be expressed in a closed form as an explicit function of system parameters as shown by Sinha \& Juneja (1991) for the case of Mathieu equation.

In order to compute the L-F transformation matrix, $L(t)$, one needs to find the STM $\Phi(t)$ associated with the linear system given by (5). If $\mathbf{A}(\mathbf{t})$ in (5) is commutative, then L(t) can be computed as

$$
\mathbf{L}(t)=e^{\mathbf{B}_{T}(t)}
$$


where $\mathbf{B}_{T}(t)$ can be obtained in a closed form as shown by Lukes (1982). For a general $\mathbf{A}(t)$, first, the Chebyshev polynomial expansion technique is used to compute the STM $\Phi(t)$. In this technique, the solution vector $\mathbf{x}(t)$ and the periodic matrix $\mathbf{A}(t)$ in equation (5) are expanded in terms of the shifted Chebyshev polynomials in the interval $[0, T]$ as shown below.

$$
\begin{aligned}
& x_{i}(t) \approx \sum_{r=0}^{s-1} b_{r}^{i} s_{r}^{*}(t) \equiv \mathbf{s}^{* T}(t) \mathbf{b}^{i}, \quad i=1,2, \ldots, n \\
& \mathbf{A}(t) \approx \sum_{r=0}^{s-1} d_{r}^{i j} s_{r}^{*}(t) \equiv \mathbf{s}^{* T}(t) \mathbf{d}^{i j}, \quad i, j=1,2, \ldots, n
\end{aligned}
$$

where $b_{r}^{i}$ are unknown expansion coefficients of $x_{i}(t), d_{r}^{i j}$ are known expansion coefficients of $A_{i j}(t)$ and $s_{r}^{*}(t)$ are the shifted Chebyshev polynomials of the first kind. For convenience in algebraic manipulation an $n \times n m$ Chebyshev polynomial matrix is defined as

$$
\hat{\mathbf{S}}(t)=\mathbf{I} \otimes \mathbf{s}^{* \mathbf{T}}(t),
$$

where $\otimes$ represents the Kronecker product (Sinha \& Wu 1991), and $I$ is an $n \times n$ identity matrix. Using the definitions in (15), (16) and (17), $x(t)$ and $\mathbf{A}(t)$ can be rewritten as

$$
\begin{aligned}
& \mathbf{x}(t)=\hat{\mathbf{S}}(t) \overline{\mathbf{b}}, \mathbf{A}(t)=\hat{\mathbf{S}}(t) \mathbf{D} \\
& \mathbf{A}(t) \mathbf{x}(t)=\hat{\mathbf{S}}(t) \overline{\mathbf{Q}} \overline{\mathbf{b}}
\end{aligned}
$$

where $\overline{\mathbf{b}}=\left\{\mathbf{b}^{1} \mathbf{b}^{2} \mathbf{b}^{3} \cdots \mathbf{b}^{n}\right\}^{T}$ is an $n m \times 1$ vector, $\mathbf{D}=\left[\mathbf{d}^{i 1} \mathbf{d}^{i 2} \mathbf{d}^{i 3} \cdots \mathbf{d}^{i j}\right], \mathbf{i j}=1,2,3$, $4, \ldots n$, is an $n m \times n$ matrix and $\overline{\mathbf{Q}}$ is an $n m \times n m$ product operation matrix (for details see Sinha \& Wu 1991).

Substituting equations (18) and (19) in the integral form of equation (5), the unknown constant $\mathbf{b}$ can be determined by a set of linear algebraic equations. Therefore, the solution vector $\mathbf{x}$ can be determined from equation (15). However, the computation of $\Phi(t)$ requires a set of solutions of (5) with $n$ initial conditions: $\mathbf{x}_{i}(0)=(1,0,0, \ldots, 0),(0,1,0, \ldots, 0)$, $(0,0,1,0, \ldots, 0), \ldots,(0,0, \ldots, 1)$. By defining the resulting set of Chebyshev coefficient vector $\overline{\mathbf{b}}_{i}^{\prime} s$ of the $n$ solutions in a matrix form, the STM can be written as (see Sinha \& Juneja 1991; Sinha \& Wu 1991; Joseph et al 1993; Sinha et al 1993; Wu \& Sinha 1994),

$$
\Phi(t)=\hat{\mathbf{S}}(t) \overline{\mathbf{B}},
$$

where $\overline{\mathbf{B}}=\left[\overline{\mathbf{b}}_{1} \overline{\mathbf{b}}_{2} \overline{\mathbf{b}}_{3} \cdots \overline{\mathbf{b}}_{n}\right]$ and $\Phi(0)=\mathbf{I}$. It has to be noted that the STM is valid only for $0 \leq t \leq T$ since the shifted Chebyshev polynomials of the first kind are defined over the interval $[0, T]$. When $t>T$, the STM can be evaluated using Floquet theory (Yakubovitch \& Starzinskii 1975) as

$$
\Phi(t)=[\Phi(\varsigma)][\Phi(T)]^{n} ; \quad t=\varsigma+n T, \quad \varsigma \epsilon[0, T], \quad n=1,2, \ldots, \epsilon .
$$

Once $\Phi(t)$ is known, the $T$-periodic complex matrix $\mathrm{L}(t)$ or the $2 T$-periodic real matrix $\mathbf{Q}(t)$ can be computed in the following way (Pandiyan et al 1993; Sinha \& Joseph 1994; Sinha \& Pandiyan 1994; Pandiyan \& Sinha 1995). Since $\Phi(0)=\mathbf{I}, \mathbf{L}(0)=\mathbf{L} T=\mathbf{I}$, the Floquet Transition Matrix (FTM) $\Phi(T)$ can be written as 


$$
\Phi(T)=e^{C T},
$$

where $\mathrm{C}$ is a $n \times n$ constant complex matrix. By performing an eigenanalysis on $\Phi(T)$, the matrix $\mathbf{C}$ can be computed easily. Then the $T$-periodic $\mathrm{L}-\mathrm{F}$ transformation matrix is

$$
\mathbf{L}(t)=\Phi(t) e^{-C t}
$$

In order to evaluate the $2 T$-periodic real L-F transformation matrix $\mathbf{Q}(\mathbf{T})$, first we note that ( $c f$ Yakubovitch \& Starzinskii 1975),

$$
\Phi(2 T)=\Phi^{2}(T)=e^{C T} e^{C^{*} T}=e^{2 \mathbf{R} T},
$$

where $\mathbf{C}^{*}$ is the conjugate matrix of $\mathbf{C}$, the $n \times n$ constant real matrix $\mathbf{R}=\left[\mathbf{C}+\mathbf{C}^{*}\right] / 2$ and the 2-T periodic L-F matrix can be represented as

$$
\begin{aligned}
\mathbf{Q}(t) & =\Phi(t) e^{-\mathbf{R} t} ; 0 \leq t \leq T ; \\
\mathbf{Q}(\tau+T) & =\Phi(\tau) \mathbf{Q}(T) e^{-\overline{\mathbf{R}} \tau} ; T \leq(T+\tau) \leq 2 T ; 0 \leq \tau \leq T .
\end{aligned}
$$

It should be noted that $\mathbf{Q}(t)=\mathbf{Q}(t+2 T)$.

If one is interested in finding $\mathbf{L}^{-1}(t)$ or $\mathbf{Q}^{-1}(t)$, then there are two avenues. $\mathbf{L}(t)$ and $\mathbf{Q}(t)$ may possibly be inverted through the use of a symbolic software like MACSYMA/ MATHEMATICA/MAPLE. However, this is neither realistic or even possible at this point in time. The other approach is to first find the STM $\Psi(t)$ of the adjoint system

$$
\dot{w}=-\mathbf{A}^{T}(t) w,
$$

and use the following relationship ( $c f$ Yakubovitch \& Starzinskii 1975),

$$
\boldsymbol{\Phi}^{-1}(t)=\Psi^{T}(t)
$$

The computation of $\Phi^{-1}(t)$ is critical in determining $\mathbf{L}^{-1}(t)$ or $\mathbf{Q}^{-1}(t)$. For example, $\mathbf{L}^{-1}(t)$ can be evaluated utilizing the properties of the adjoint system as shown below,

$$
\mathbf{L}^{-1}(t)=\left[\boldsymbol{\Phi}(t) e^{-C t}\right]^{-1}=e^{C t} \boldsymbol{\Phi}^{-1}(t)=e^{C t} \boldsymbol{\Psi}^{T}(t) .
$$

Such an approximation of $\mathrm{L}-\mathrm{F}$ transformations has been found to be extremely convergent (Pandiyan et al 1993; Sinha \& Joseph 1994; Sinha \& Pandiyan 1994; Pandiyan \& Sinha $1995)$ and since it is periodic with period $T$, the elements $\mathbf{L}_{i j}(t)$ and $\mathbf{Q}_{i j}(t)$ have the truncated Fourier representations

$$
\begin{aligned}
& \mathbf{L}_{i j}(t) \approx \sum_{n=-q}^{q} c_{n} \exp (i 2 \pi n t / T), \quad i=\sqrt{-1} \\
& \mathbf{Q}_{i j}(t) \approx \frac{a_{0}}{2}+\sum_{n=1}^{q} a_{n} \cos \frac{\pi n t}{T}+\sum_{n=1}^{q} b_{n} \sin \frac{\pi n t}{T}
\end{aligned}
$$

respectively.

Since complex matrix $\mathbf{L}(\mathbf{t})$ (or the real matrix $\mathbf{Q}(\mathbf{t})$ ) can be computed as a function of $t$, all algebraic manipulations involving this matrix can be done in symbolic form using MATHEMATICA or MACSYMA. $\mathbf{L}_{i j}^{-1}(t)$ and $\mathbf{Q}_{i j}^{-1}(t)$ have similar Fourier representations.

In this study only the real L-F transformation $\mathbf{Q}(\mathbf{t})$ has been used to make it more appealing to the engineering community. A 12- to 15-term Chebyshev expansion has been found to yield extremely accurate representation of $Q(t)$. The accuracy of $Q(t)$ is directly dependent upon the convergence and accuracy of the STM $\Phi(t)$ itself. A convergence study in the computation of $\Phi(\mathbf{t})$ has been reported by Joseph et al (1993). 


\section{Time-dependent normal forms and centre manifold reduction}

\subsection{Normal forms}

The fact that $\mathbf{A}(t)$ is time dependent in (4), a direct application of normal form theory is not possible. Using the transformation

$$
\mathbf{x}(t)=\mathbf{Q}(t) z(t),
$$

(4) takes the form

$$
\dot{z}=\mathbf{R} z+\mathbf{Q}^{-1}(t)\left\{\mathbf{f}_{2}(z, t)+\mathbf{f}_{3}(z, t)\right\},
$$

where $\mathbf{R}$ is an $n \times n$ constant matrix and nonlinear terms of order four and higher have been neglected, since for generic codimension 1 bifurcations, the fourth order terms do not affect the local stability behaviour (Arnold 1988). The form of (32) is amenable to direct application of the method of time dependent normal forms (TDNF) for equations with periodic coefficients as shown by Arnold (1988).

Equation (32) in its Jordan canonical form can be written as

$$
\dot{y}=\mathbf{J} y+w_{2}(y, t)+w_{3}(y, t)
$$

where $\mathbf{J}$ is the Jordan form of matrix $\mathbf{R}$ and $w_{k}(y, t)$ are $2 T$-periodic functions and contain homogeneous monomials of $y_{i}$ of order 2 and 3 . Using a sequence of near identity transformations of the form

$$
y=v+h_{r}(v, t)
$$

where $h_{r}(v, t)$ is a formal power series in $v$ of degree $r(r=2,3)$ with periodic coefficients having the principal period $2 T$, (33) can be reduced to its simplest form

$$
\dot{v}=\mathbf{J} v+w_{2}(v, t)+w_{3}(v, t) .
$$

It is important to note that the $w_{2}(\nu, t)$ and $w_{3}(\nu, t)$ contain only a finite number of Fourier harmonics. This is due to the fact that the solution of the resulting homological equation depends on the resonance condition relating the eigenvalues of $\mathbf{J}$ and the Fourier frequencies of $\mathbf{w}_{r}(\nu, t)$ (Arnold 1988). It should be pointed out that the solution of the time-dependent homological equation requires the solution of a large set of linear algebraic equations even for a $2 \times 2$ system. For example, if for such a system, the $L-F$ transformation matrix $\mathbf{Q}(t)$ is represented by a fifteen-term complex Fourier expansion and let us say that the degree of the monomials $r=3$, then one needs to solve $(2 \times 124)$ equations in blocks of 31 .

\subsection{Centre manifold reduction}

In situations where some of the eigenvalues of $\mathbf{J}$ in (33) are critical, the stability of (33) can be discussed in the centre manifold via time-periodic centre manifold theorems. Application of the normal form procedure to the reduced set of equations in the centre manifold is found to retain the stability characteristics of the original $n$-dimensional system. In the 
following, a theorem due to Malkin (1962) has been utilized to develop a practical method for finding the centre manifold relations for the time-periodic systems.

Let us assume that (33) has $n_{1}$ eigenvalues that are critical and $n_{2}$ eigenvalues that have negative real parts. Therefore, (33) may be rewritten in the form

$$
\left\{\begin{array}{l}
\dot{y}_{c} \\
\dot{y}_{s}
\end{array}\right\}=\left[\begin{array}{cc}
\mathbf{J}_{c} & 0 \\
0 & \mathbf{J}_{s}
\end{array}\right]\left\{\begin{array}{l}
y_{c} \\
y_{s}
\end{array}\right\}+\left\{\begin{array}{l}
w_{c 2} \\
w_{s 2}
\end{array}\right\}+\left\{\begin{array}{l}
w_{c 3} \\
w_{s 3}
\end{array}\right\}
$$

where the subscripts $c$ and $s$ represent the critical and stable vectors, respectively. According to the centre manifold theorem, there exists a relation (Malkin 1962; Pandiyan \& Sinha 1995)

$$
y_{s}=h\left(y_{c}, t\right),
$$

such that $h\left(y_{c}, t\right)$ is of the form,

$$
h\left(y_{c}, t\right)=\sum B_{s}^{\left(m_{1} \cdots m_{n_{1}}\right)}(t) y_{1}^{m_{1}} \cdots y_{n_{1}}^{m_{n_{1}}} ; m_{1}+\cdots+m_{n_{1}} \geq 1,
$$

where $B_{s}^{m_{1} \cdots m_{n_{1}}}(t)$ are periodic coefficients with period $2 T$. The relation $y_{s}$ given by equation (32) can be obtained as the formal solutions of the equations (see Malkin 1962; Pandiyan \& Sinha 1995)

$$
\frac{\partial y_{s}}{\partial t}+\sum_{i=1}^{n_{1}} \frac{\partial y_{s}}{\partial y_{c}}\left(\mathbf{J}_{s} y_{s}+\mathbf{W}_{s}\right)=\mathbf{J}_{c} y_{c}+\mathbf{W}_{c}
$$

where $\mathbf{W}_{c}=w_{c 2}+w_{c 3}$ and $\mathbf{W}_{s}=w_{s 2}+w_{s 3}$ are nonlinear vector monomials of the critical and stable states of the system, respectively. It is important to note that the resulting solutions will be meaningful only if the coefficients $B_{s}^{m_{1} \cdots m_{n_{1}}}(t)$ are also periodic. Although there exists an infinite number of expansions similar to (38) which have finite coefficients and also satisfy (39), there is only one with periodic coefficients. This result was first reported by Malkin (1962).

As a result of substitution of (38) in (39), a set of differential equations in terms of the unknown coefficients $B_{s}^{m_{1} \cdots m_{n_{1}}}(t)$ is obtained in a form

$$
\frac{\mathrm{d} B_{s}^{\left(m_{1} \cdots m_{n_{1}}\right)}}{\mathrm{d} t}-\lambda_{j} B_{s}^{\left(m_{1} \cdots m_{n_{1}}\right)}=C_{s}^{\left(m_{1} \cdots m_{n_{1}}\right)}
$$

where $\lambda_{j}, j=1,2, \ldots, n_{2}$ are the eigenvalues of the stable part of the system and $C_{s}^{\left(m_{1} \cdots m_{n_{1}}\right)}$ are the known integral rational functions of the periodic coefficients on the right hand side of (40). The coefficients $B_{s}^{\left(m_{1} \cdots m_{n_{1}}\right)}$ can be obtained by formally solving the above set of differential equations. For this purpose, $B_{s}^{\left(m_{1} \cdots m_{n_{1}}\right)}$ is assumed in the form of a finite Fourier expansions as

$$
\mathbf{B}_{s}^{\left(m_{1} \cdots m_{n_{1}}\right)}(t)=a_{0}+\sum_{n=1}^{l} a_{n} \cos \left(\frac{2 \pi n t}{\hat{T}}\right)+b_{n} \sin \left(\frac{2 \pi n t}{\hat{T}}\right)
$$

where $\hat{T}=2 T$. Substituting (41) in (40) and equating like terms on both sides of the equation, a set of algebraic equations in terms of the unknown coefficients $a_{n}$ and $b_{n}$ are 
obtained. The constants $a_{n}$ and $b_{n}$ can be computed by solving these algebraic equations and therefore the coefficients $B_{s}^{\left(m_{1} \cdots m_{n_{1}}\right)}$ can be determined in the form of (41). Substitution of (32) in (36) clearly decouples the stable and critical states and, hence, the problem reduces to the investigation of stability of an $n_{1}$ dimensional system in the centre manifold. The resulting system of $n_{1}$ periodic equations is of the form

$$
\dot{\mathbf{y}}_{c}=\mathbf{J}_{c} \mathbf{y}_{c}+\mathbf{W}_{c}^{*}
$$

where vector $\mathbf{W}_{c}^{*}$ contains nonlinear monomials which are functions of $\mathbf{y}_{c}$ only.

\section{Applications}

To demonstrate the applicability and effectiveness of the suggested approach, two examples are considered. As a first example, a nonlinear Mathieu equation is considered for which the $\mathrm{L}-\mathrm{F}$ transformation has been obtained using the computational algorithm discussed in $\S 3$. Although this is a simple example, it brings out the key points clearly and shows the superiority of the proposed methods over the classical methods such as averaging, perturbation, etc. Whereas the results of the proposed technique, based on L-F transformation and normal forms, provide reasonably good solutions even for moderately large parameters multiplying the nonlinear terms, the traditional averaging procedure is applicable only when the parameters multiplying the periodic terms and the nonlinear terms are both small.

In the second example, the dynamic behavior of a time-periodic double pendulum undergoing various bifurcations is examined. In particular the critical dynamics under secondary Hopf and flip bifurcations are studied in detail.

\subsection{Example 1. Mathieu equation with cubic nonlinearity}

Consider the Mathieu equation with cubic nonlinearity in the form,

$$
\ddot{x}+\delta \dot{x}+(\alpha+\beta \cos \omega t) x+\epsilon x^{3}=0,
$$

where $\delta, \alpha, \beta, \omega$ and $\epsilon$ are the parameters of the system. In the state space form the above equation is rewritten as

$$
\left\{\begin{array}{l}
\dot{x}_{1} \\
\dot{x}_{2}
\end{array}\right\}=\left[\begin{array}{cc}
0 & 1 \\
-(\alpha+\beta \cos \omega t) & -\delta
\end{array}\right]\left\{\begin{array}{l}
x_{1} \\
x_{2}
\end{array}\right\}+\left\{\begin{array}{c}
0 \\
-\epsilon x_{1}^{3}
\end{array}\right\}
$$

where $\left\{\dot{x}_{1}, \dot{x}_{2}\right\}^{T}=\{x, \dot{x}\}^{T}$. Following the steps described in $\S 3$, the $2-T$ periodic real $\mathrm{L}-\mathrm{F}$ transformation matrix $\mathrm{Q}(t)$ can be computed for a given parameter set. Applying the transformation $\mathbf{x}=\mathbf{Q}(t) z$, (44) is transformed to

$$
\left\{\begin{array}{l}
\dot{z}_{1} \\
\dot{z}_{2}
\end{array}\right\}=\left[\begin{array}{ll}
R_{11} & R_{12} \\
R_{21} & R_{22}
\end{array}\right]\left\{\begin{array}{l}
z_{1} \\
z_{2}
\end{array}\right\}+\mathbf{Q}^{-1}(t)\left\{\begin{array}{c}
0 \\
-\epsilon\left(Q_{11} z_{1}+Q_{12} z_{2}\right)^{3}
\end{array}\right\},
$$

where $R_{i j}$ are the elements of the real matrix $\mathbf{R}$ and $Q_{i j}$ are the elements of the L-F transformation matrix $\mathbf{Q}(t)$. It is to be noted that the nonlinear part of (45) can be expressed in 
Fourier form due to the periodic nature of the Liapunov-Floquet transformation. Equation (45) can be written in the canonical form as

$$
\begin{aligned}
&\left\{\begin{array}{l}
\dot{y}_{1} \\
\dot{y}_{2}
\end{array}\right\}=\left[\begin{array}{cc}
\lambda_{1} & 0 \\
0 & \lambda_{2}
\end{array}\right]\left\{\begin{array}{l}
y_{1} \\
y_{2}
\end{array}\right\} \\
&+\epsilon\left\{\begin{array}{l}
f_{11}(t, \tau) y_{1}^{3}+f_{12}(t, \tau) y_{1}^{2} y_{2}+f_{13}(t, \tau) y_{1} y_{2}^{2}+f_{14}(t, \tau) y_{2}^{3} \\
f_{21}(t, \tau) y_{1}^{3}+f_{22}(t, \tau) y_{1}^{2} y_{2}+f_{23}(t, \tau) y_{1} y_{2}^{2}+f_{24}(t, \tau) y_{2}^{3}
\end{array}\right\}
\end{aligned}
$$

where $\tau=2 T, \Lambda=\left\{\lambda_{1}, \lambda_{2}\right\}$ are the eigenvalues of $\mathbf{R}$ and the periodic coefficients $f_{i j}(t, \tau) ; i, j=1, \ldots, 4$ are expressed as

$$
f_{i j}(t, \tau)=a_{0}^{i, j}+\sum_{n=1}^{l} a_{n}^{i, j} \cos \left(\frac{2 \pi n t}{\tau}\right)+\sum_{n=1}^{l} b_{n}^{i, j} \sin \left(\frac{2 \pi n t}{\tau}\right) .
$$

After experimenting with various sets of system parameters, it was observed that $l=15$ provided quite accurate representations of functions $f_{i j}(t, \tau)$. This has been reported earlier by Pandiyan et al (1993). It is also consistent with the number of Fourier terms taken in the representation of the L-F transformation $\mathbf{Q}(t)$.

In order to obtain a solution of equation (46) using TDNF, consider a near-identity nonlinear transformation

$$
\begin{aligned}
& y_{1}=u+g_{11}(t, \tau) u^{3}+g_{12}(t, \tau) u^{2} v+g_{13}(t, \tau) u v^{2}+g_{14}(t, \tau) v^{3}, \\
& y_{2}=v+g_{21}(t, \tau) u^{3}+g_{22}(t, \tau) u v^{2}+g_{23}(t, \tau) u v^{2}+g_{24}(t, \tau) v^{3},
\end{aligned}
$$

where the periodic coefficients $g_{i j}(t, \tau) ; i, j=1, \ldots, 4$ are once again of the form given by (47) but with unknown constants $\tilde{a}_{n}$ and $\tilde{b}_{n}$. Substituting (48) in (46) and solving the resulting homological equation as described earlier, the unknown constants $\tilde{a}_{n}$ and $\tilde{b}_{n}$ can be evaluated. In situations when there is no resonance, the Fourier series assumed for $g_{i j}(t, \tau)$ and its derivative are found to be convergent (Arnold 1988). On the other hand, if resonance takes place, the unknown constants of the corresponding periodic coefficient cannot be determined and some nonlinear terms remain even after the normal form reduction. As long as there is no resonance, all nonlinear terms are eliminated and the reduced normal form is just the linear part of (46). Therefore, the solution of the nonlinear Mathieu equation in the original coordinates can be obtained by substituting back all the intermediate transformations. Even when some of the nonlinear terms remain due to resonance, the resulting equation can still be used to provide many useful conclusions about the stability and dynamical behaviour of the system. Such procedures are described by Bruno (1989) and Hale \& Kocak (1991).

In order to obtain an approximate solution of (46) via the time independent normal form (TINF) theory, variations of the periodic coefficients of nonlinear terms are neglected in comparison with their predominant means. This approximation results in an equation of the form

$$
\left\{\begin{array}{l}
\dot{y}_{1} \\
\dot{y}_{2}
\end{array}\right\}=\left[\begin{array}{cc}
\lambda_{1} & 0 \\
0 & \lambda_{2}
\end{array}\right]\left\{\begin{array}{l}
\tilde{y}_{1} \\
\tilde{y}_{2}
\end{array}\right\}+\left\{\begin{array}{l}
a_{3} \tilde{y}_{1}^{3}+a_{4} \tilde{y}_{1}^{2} \tilde{y}_{2}+a_{5} \tilde{y}_{1} \tilde{y}_{2}^{2}+a_{6} \tilde{y}_{2}^{3} \\
b_{3} \tilde{y}_{1}^{3}+b_{4} \tilde{y}_{1}^{2} \tilde{y}_{2}+b_{5} \tilde{y}_{1} \tilde{y}_{2}^{2}+b_{6} \tilde{y}_{2}^{3}
\end{array}\right\},
$$


where $\tilde{y}_{1}$ and $\tilde{y}_{2}$ are the approximate states and $a_{j}, b_{j}, j=3, \ldots, 6$ are the means of the periodic coefficients of (47). To apply the TINF theory, consider a nonlinear near-identity transformation of the form

$$
\begin{aligned}
& \tilde{y}_{1}=u+p_{1} u^{3}+p_{2} u^{2} v+p_{3} u v^{2}+p_{4} v^{3}, \\
& \tilde{y}_{2}=v+q_{1} u^{3}+q_{2} u^{2} v+q_{3} u v^{2}+q_{4} v^{3},
\end{aligned}
$$

where $p_{i}$ and $q_{i}$ are unknown constants. Substituting (50) in (49) and solving the resulting autonomous homological equation, (49) can be reduced to a linear form in most of the situations except when resonances due to nonlinearity occur. The approximate solutions for this case can also be obtained in a fashion similar to the procedure discussed above. It is also observed that as long as the system has eigenvalues that are distinct with negative or positive real parts, one could completely reduce the system to a linear form.

At this point an application of the traditional averaging procedure to (43) is briefly discussed. Assume a generating solution of the form ( $c f$ Sanders \& Verhulst 1985),

$$
x(t)=z_{1}(t) \cos \omega_{0} t+\frac{z_{2}(t)}{\omega_{0}} \sin \omega_{0} t
$$

where $\omega_{0}^{2}=\alpha$ and $z_{i}, i=1,2$ are the slowly varying coefficients of the solution. Using this solution in (43) and averaging over the principal period $2 \pi / \omega$, one gets a set of quasilinear autonomous differential equations with cubic nonlinearity in the averaged coefficients $\tilde{z}$ as

$$
\left\{\begin{array}{l}
\dot{z}_{1} \\
\dot{z}_{2}
\end{array}\right\}=\left[\begin{array}{cc}
R_{11}^{0} & R_{12}^{0} \\
R_{21}^{0} & R_{22}^{0}
\end{array}\right]\left\{\begin{array}{l}
\tilde{z}_{1} \\
\tilde{z}_{2}
\end{array}\right\}+\epsilon\left\{\begin{array}{l}
\bar{a}_{3} \tilde{z}_{1}^{3}+\bar{a}_{4} \tilde{z}_{1}^{2} \tilde{z}_{2}+\bar{a}_{5} \tilde{z}_{1} \tilde{z}_{2}^{2}+\bar{a}_{6} \tilde{z}_{2}^{3} \\
\bar{b}_{3} \tilde{z}_{1}^{3}+\bar{b}_{4} \tilde{z}_{1}^{2} \tilde{z}_{2}+\bar{b}_{5} \tilde{z}_{1} \tilde{z}_{2}^{2}+\bar{b}_{6} \tilde{z}_{2}^{3}
\end{array}\right\},
$$

where $\bar{a}_{i}$ and $\bar{b}_{i}, i=3, \ldots, 6$ are the constants depending on the parameters of the system and $R_{i j}^{0}$ are the elements of the constant matrix $\mathbf{R}^{0}$. However, the eigenvalues of the matrix $\mathbf{R}^{0}$ will be quite different from those appearing in (49). The solution of the above equation can be discussed via the TINF theory.

\subsection{Case studies}

In the following, several case studies are presented by selecting various sets of parameters $\alpha, \beta, \delta$ and $\epsilon$ in the Mathieu equation given by (43). The parameter $\omega$ is selected as $2 \pi$ in all cases of this study.

Case 1. Trajectories in stable manifold $(\alpha \neq 0)$ : Parameter set $1: \alpha=10.0 ; \beta=0.2$; $\delta=1.8974 ; \epsilon=0.3$; Note that the parameters $\beta$ and $\epsilon$ multiplying the periodic and the nonlinear terms respectively are selected small. The suggested approaches, including the traditional averaging, are applied to this set and the results, along with the numerical solution, are presented in figure 1 . It is understandable that all the methods predict the behaviour of the system correctly due to the smallness of the parameters $\beta$ as well as $\epsilon$. For brevity, the coefficients of near-identity transformations for the normal forms are not recorded in this paper.

Parameter set $2: \alpha=0.5 ; \beta=4.0 ; \delta=0.4243 ; \epsilon=0.3$; For this case, $\beta$, the parameter multiplying the periodic term, is selected to be 8 times larger than $\alpha$. From figure 2 , 


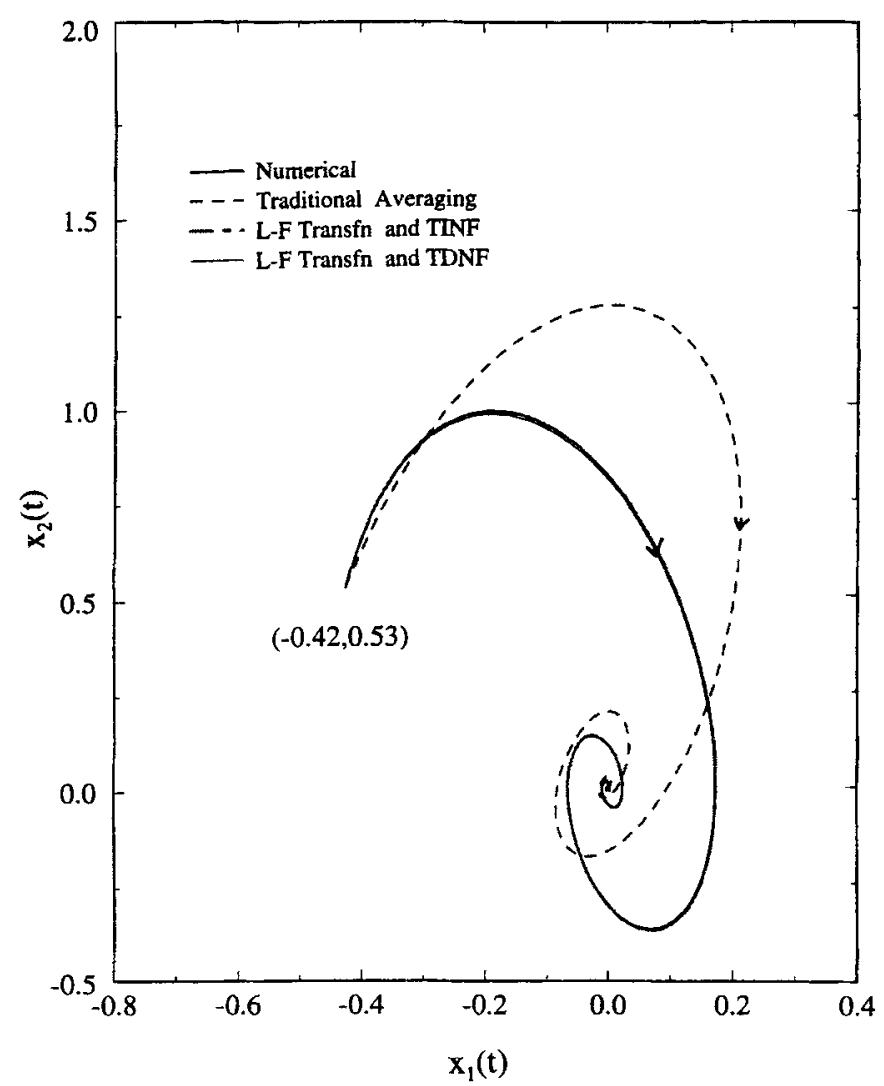

Figure 1. Comparison of solutions of Mathieu's equation with cubic nonlinearity: $\alpha=10$, $\beta=0.2, \delta=1.8974, \epsilon=0.3$.

it is observed that except for the averaging method, all other techniques predict similar trajectories which finally approach the fixed point $(0,0)$.

Case 2. Equation without generating solution $(\alpha=0)$ : Note that when $\alpha=0$ the fundamental frequency $\omega_{0}$ of the autonomous part of (43) is zero and without.a generating solution the averaging approach cannot be applied.

Parameter set: $\alpha=0 ; \beta=4.0 ; \delta=0.4243 ; \epsilon=3.0$; This set shows that the behaviour of the system is well predicted by the suggested techniques when compared with the numerical solution even though the nonlinearity parameter $\epsilon$ is 10 times larger than the value used in set 1 . The comparison is shown in figure 3 .

The coefficients of the near-identity transformations for the TDNF as well as TINF methods are once again omitted for brevity.

Case 3. The centre manifold case: The application of the suggested techniques to the special case when the resonance condition prevails due to the presence of a pair of purely imaginary roots is shown in this part. For this case, by applying normal forms, all the non-resonant terms are annihilated, however, the homological equation corresponding to the resonant terms cannot be resolved. Therefore, some of the nonlinear terms of third 


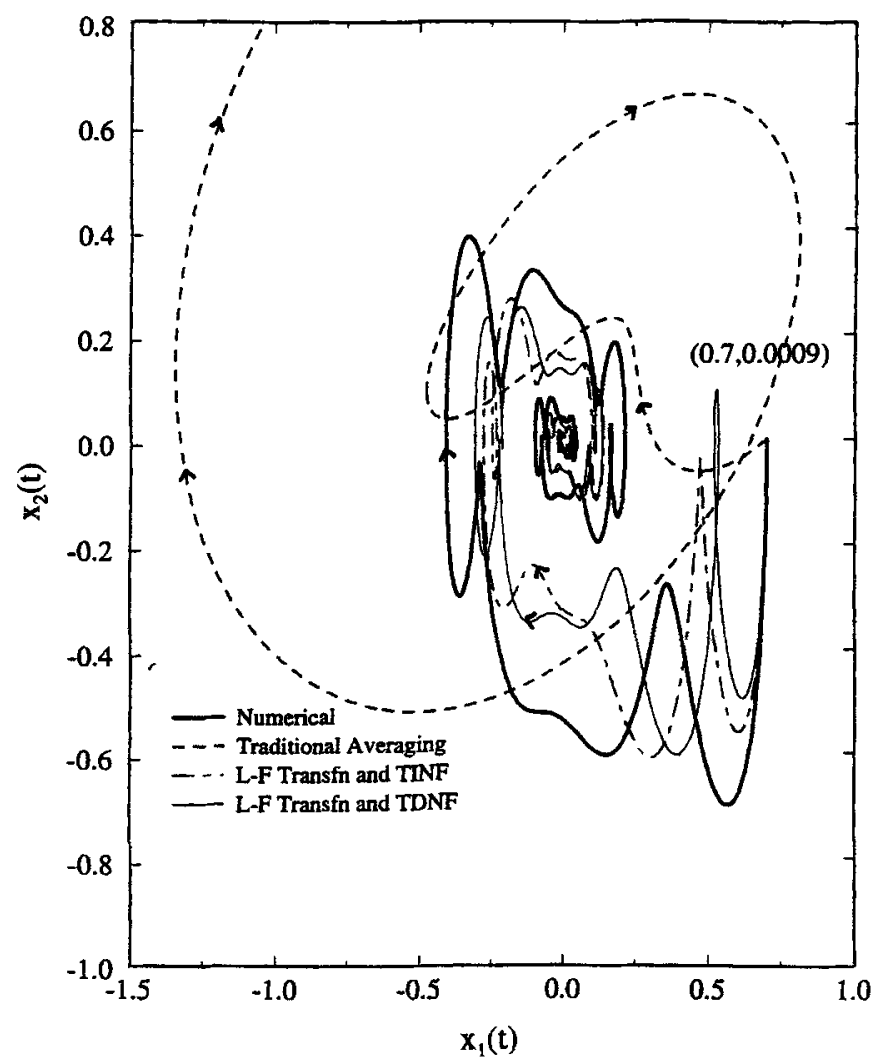

Figure 2. Comparison of solutions of Mathieu's equation with cubic nonlinearity: $\alpha=0.5$, $\beta=4.0, \delta=0.4243, \epsilon=0.3$.

degree stay in the reduced equation and hence, in general, a closed form solution is not possible. Under such circumstances it may be possible to neglect the periodic variations of the nonlinear coefficients and still retain the stability behaviour of the system. In the following, a case study is provided to demonstrate the applications in similar situations. Parameter set: $\alpha=4.0 ; \beta=7.468 ; \delta=0 ; \epsilon=0.3$; For this set of parameters, applications of the L-F transformation, the Jordan canonical transformation and the near-identity transformation ( $c f(48))$ to (46) results in an equation of the form,

$$
\left\{\begin{array}{l}
\dot{u} \\
\dot{v}
\end{array}\right\}=\left[\begin{array}{cc}
-i \omega_{0} & 0 \\
0 & i \omega_{0}
\end{array}\right],\left\{\begin{array}{l}
u \\
v
\end{array}\right\}+\epsilon\left\{\begin{array}{l}
f_{12}(t, \tau) u^{2} v \\
f_{23}(t, \tau) u v^{2}
\end{array}\right\},
$$

where, $f_{12}(t, \tau)$ and $f_{23}(t, \tau)$ are complexified periodic functions corresponding to the resonant terms and $\pm i \omega_{0}= \pm i 0.816$ are the eigenvalues of the system. Multiplying $\dot{u}$ by $v$ and $\dot{v}$ by $u$ and adding, a linear differential equation in $(u v)$ can be obtained. Therefore, the analytical solution of (53) can be found in $(u v)$ which is of the form

$$
u v=-1 / \int_{0}^{t} a(\chi, \tau) \mathrm{d} \chi
$$

where $a(t, \tau)$ is a complex Fourier function. The differential equation (53) can be decoupled by substituting the solution (54) into (53) and the resulting linear differential equation in 


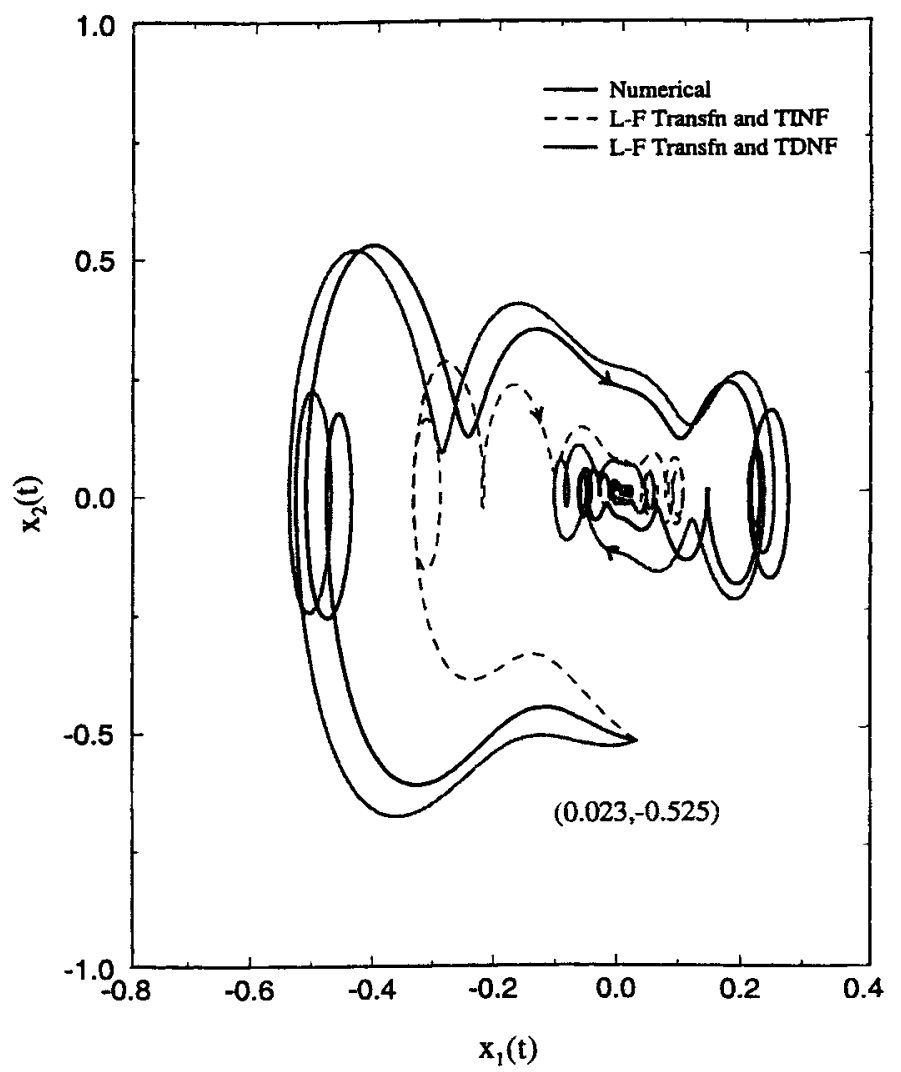

Figure 3. Comparison of solutions of Mathieu's equation with cubic nonlinearity: $\alpha=0$, $\beta=4.0, \delta=0.18974, \epsilon=0.3$.

$u$ can be shown to be of the form,

$$
\begin{aligned}
& \dot{u}=\left[-i \omega_{0}+\epsilon f_{12}(t, \tau) /\left(-\int_{0}^{t} a(\chi, \tau) \mathrm{d} \chi\right)\right] u, \\
& \dot{u}=\left[-i \omega_{0}+\epsilon \sum_{n=-q}^{q} c_{n} \exp (i 2 \pi n t / \tau)\right] u,
\end{aligned}
$$

where $c_{n}$ are complex constants of Fourier expansion of the periodic terms and hence

$$
u=\exp \left[\left(-i \omega_{0}+\epsilon \sum_{n=-q}^{q} c_{n} \exp (i 2 \pi n t / \tau)\right) t\right] u_{0}
$$

In a similar manner, $v$ can also be computed. It should be noted that the stability of these solutions entirely depend on the real part of the constant coefficient of the time-varying function $c_{n} \exp (i 2 \pi n t / \tau)$. The solution is stable and/or unstable depending on whether the constant has negative real part or positive real part, respectively. This observation has also been made by Rosenblat \& Cohen $(1980,1981)$ following an entirely different approach. When the real part is zero, the solutions are closed orbits and behave like limit cycles. A typical result is shown in figure 4. 


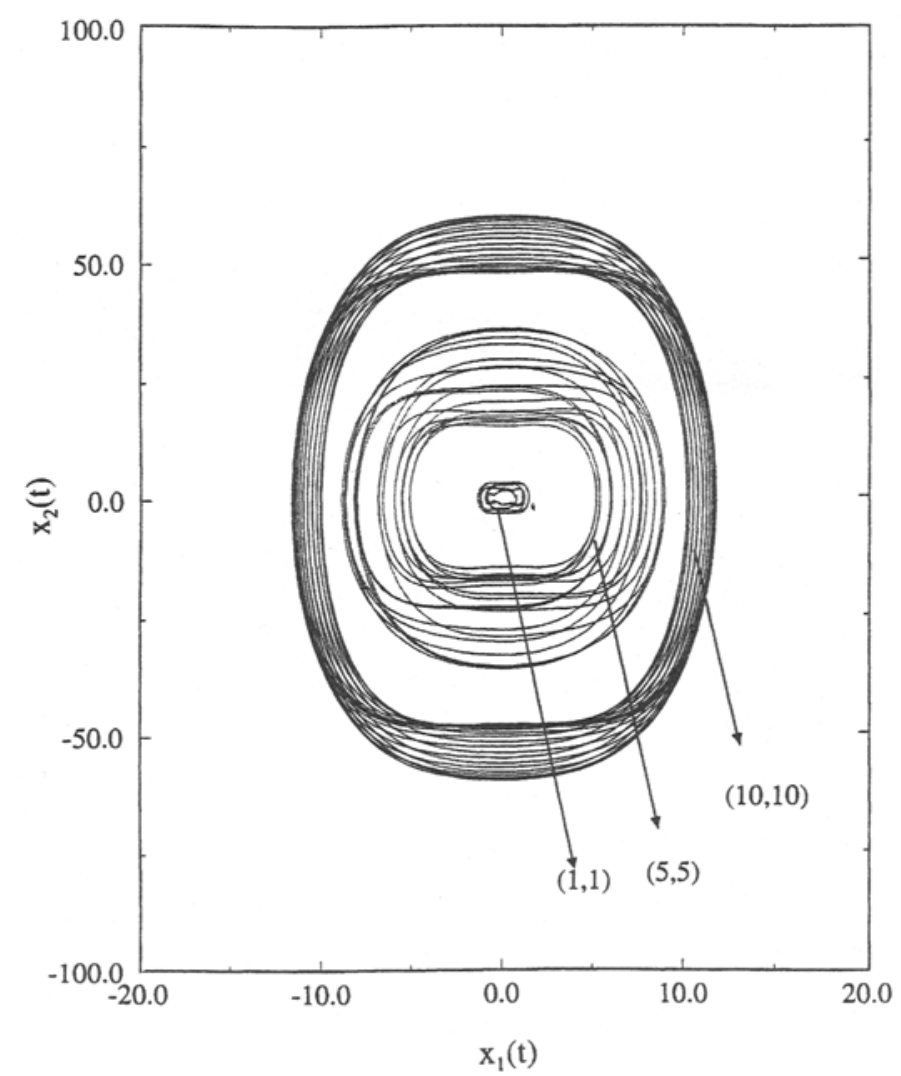

Figure 4. Phase plots of Mathieu's equation in centre manifold: $\alpha=4.0, \beta=7.468, \delta=0, \epsilon=0.3$.

\section{Example 2. Double inverted pendulum with parametric excitation}

In this example, the bifurcation of a two-mass inverted pendulum subjected to nonconservative periodic load is discussed. The nonlinear equations of motion of the system are of the form (Jin \& Matsuzaki 1988; Pandiyan \& Sinha 1995)

$$
\begin{aligned}
\ddot{\phi}_{1}= & -0.5\left(B_{1}+2 B_{2}\right) \dot{\phi}_{1}+B_{2} \dot{\phi}_{2}+0.5 \bar{k}(\bar{p}-3) \phi_{1} \\
& +0.5 \bar{k}(2-\bar{p}) \phi_{2}-0.5\left(\dot{\phi}_{1}^{2}+\dot{\phi}_{2}^{2}\right)\left(\phi_{1}-\phi_{2}\right) \\
& -(\bar{p} / \bar{k} / 12)\left\{\left(\phi_{1}-\gamma \phi_{2}\right)^{3}-(1-\gamma)^{3} \phi_{2}^{3}\right\}-\left(\left(\phi_{1}-\phi_{2}\right)^{2} / 4\right) \\
& \times\left\{\bar{k}(\bar{p}-4) \phi_{1}+\bar{k}(3+\bar{p}(\gamma-2)) \phi_{2}-\left(B_{1}+3 B_{2}\right) \dot{\phi}_{1}+3 B_{2} \dot{\phi}_{2}\right\} \\
\ddot{\phi}_{2}= & 0.5\left(B_{1}+4 B_{2}\right) \dot{\phi}_{1}-2 B_{2} \dot{\phi}_{2}+0.5(5-\bar{p}) \bar{k} \phi_{1} \\
& +\{(\bar{p}(1.5-\gamma)-2) \bar{k}\} \phi_{2}+0.5\left(\phi_{1}-\phi_{2}\right)\left(3 \dot{\phi}_{1}^{2}+\dot{\phi}_{2}^{2}\right) \\
& +(\bar{p} \bar{k} / 12)\left\{\left(\phi_{1}-\gamma \phi_{2}\right)^{3}-3(1-\gamma)^{3} \phi_{2}^{3}\right\}+\left(\left(\phi_{1}-\phi_{2}\right)^{2} / 4\right) \\
& \times\left\{\bar{k}(2 \bar{p}-7) \phi_{1}+\bar{k}(5+\bar{p}(\gamma-3)) \phi_{2}-\left(2 B_{1}+5 B_{2}\right) \dot{\phi}_{1}+5 B_{2} \dot{\phi}_{2}\right\}
\end{aligned}
$$

where $m$ is the mass, $l$ is the length of the links of the pendulum, $\phi_{1}$ and $\phi_{2}$ the displacement angles, $\dot{\phi}_{1}$ and $\dot{\phi}_{2}$ are the corresponding rates, $\gamma(0 \leq \gamma \leq 1)$ is the load-direction parameter and $P=P_{1}+P_{2} \cos \omega \tau$. Other symbols appearing in (57) and (58) are defined as $b_{1} \&$ $b_{2}=$ damping parameters, $B_{1}=b_{1} / m l^{2}, B_{2}=b_{2} / m l^{2}, \bar{p}=P l / m l^{2}, \bar{k}=k / m l^{2}$ 
$k=$ stiffness parameter, $P_{1}=$ magnitude of static load, $P_{2}=$ amplitude of the dynamic periodic load. Equations (57) and (58) are rewritten in the state-space form as

$$
\begin{aligned}
& \left\{\begin{array}{l}
\dot{y}_{1} \\
\dot{y}_{2} \\
\dot{y}_{3} \\
\dot{y}_{4}
\end{array}\right\}=\left[\begin{array}{cccc}
0 & 0 & 1 & 0 \\
0 & 0 & 0 & 1 \\
0.5 \bar{k}(\bar{p}-3) & 0.5 \bar{k}(2-\bar{p}) & -0.5\left(B_{1}+2 B_{2}\right) & B_{2} \\
0.5 \bar{k}(5-\bar{p}) & \bar{k}[\bar{p}(1.5-\gamma)-2] & 0.5\left(B_{1}+4 B_{2}\right) & -2 B_{2}
\end{array}\right] \\
& \times\left\{\begin{array}{l}
y_{1} \\
y_{2} \\
y_{3} \\
y_{4}
\end{array}\right\}+\left\{\begin{array}{c}
0 \\
0 \\
{\left[-0.5\left(y_{3}^{2}+y_{4}^{2}\right)\left(y_{1}-y_{2}\right)-(\bar{p} \bar{k})\left[\left(y_{1}-\gamma y_{2}\right)^{3}\right.\right.} \\
\left.-(1-\gamma)^{3} y_{2}^{3}\right] / 12-0.25\left(y_{1}-y_{2}\right)^{2}\left[\bar{k}(\bar{p}-4) y_{1}\right. \\
\left.\left.+\bar{k}(3+\bar{p}(\gamma-2)) y_{2}-\left(B_{1}+3 B_{2}\right) y_{3}+3 B_{2} y_{4}\right]\right] \\
{\left[0.5\left(y_{1}-y_{2}\right)\left(3 y_{3}^{2}+y_{4}^{2}\right)+\bar{p} \bar{k}\left[\left(y_{1}-\gamma y_{2}\right)^{3}\right.\right.} \\
\left.-3(1-\gamma)^{3} y_{2}^{3}\right] / 12+0.25\left(y_{1}-y_{2}\right)^{2}\left[\bar{k}(2 \bar{p}-7) y_{1}\right. \\
\left.\left.+\bar{k}(5+\bar{p}(\gamma-3)) y_{2}-\left(2 B_{1}+5 B_{2}\right) y_{3}+5 B_{2} y_{4}\right]\right]
\end{array}\right\}
\end{aligned}
$$

where $\left\{y_{1}, y_{2}, y_{3}, y_{4}\right\}=\left\{\phi_{1}, \phi_{2}, \dot{\phi}_{1}, \dot{\phi}_{2}\right\}$. In the following, the dynamics of a primary single Hopf and a single flip bifurcations of the above 4-dimensional system is discussed via centre manifold principle by reducing the problem to a two and a single dimension respectively.

(i) Hopf bifurcation - For the parameter set, $\bar{k}=2.0, B_{1}=B_{2}=0.016, P_{1}=0.5, P_{2}=$ $0.966, \gamma=0.8, \omega=1.0,(46)$ yields a pair of complex Floquet multipliers with modulus one which corresponds to a single Hopf bifurcation. After normalizing the time with $\omega \tau=2 \pi t$, the $\mathrm{L}-\mathrm{F}$ transformation corresponding to (59) is computed. The application of this transformation to (59) leads to the following dynamically equivalent Jordan canonical form.

$$
\begin{aligned}
& \left\{\begin{array}{l}
\dot{y}_{1} \\
\dot{y}_{2} \\
\dot{y}_{3} \\
\dot{y}_{4}
\end{array}\right\}= \\
& {\left[\begin{array}{cccc}
-0.17791+1.14613 i & 0 & 0 & 0 \\
0 & -0.17791-1.14613 i & 0 & 0 \\
0 & 0 & 0.3391 i & 0 \\
0 & 0 & 0 & -0.3391 i
\end{array}\right]} \\
& \quad \times\left\{\begin{array}{l}
y_{1} \\
y_{2} \\
y_{3} \\
y_{4}
\end{array}\right\}+\left\{\begin{array}{l}
\sum \mathbf{a}_{q}(t) y_{1}^{m_{1}} y_{2}^{m_{2}} y_{3}^{m_{3}} y_{4}^{m_{4}} \\
\sum \mathbf{b}_{q}(t) y_{1}^{m_{1}} y_{2}^{m_{2}} y_{3}^{m_{3}} y_{4}^{m_{4}} \\
\sum \mathbf{c}_{q}(t) y_{1}^{m_{1}} y_{2}^{m_{2}} y_{3}^{m_{3}} y_{4}^{m_{4}} \\
\sum \mathbf{d}_{q}(t) y_{1}^{m_{1}} y_{2}^{m_{2}} y_{3}^{m_{3}} y_{4}^{m_{4}}
\end{array}\right\}, \sum_{i=1}^{4} m_{i}=3
\end{aligned}
$$

where $\mathbf{a}_{q}(t), \mathbf{b}_{q}(t), \mathbf{c}_{q}(t)$ and $\mathbf{d}_{q}(t)$ are the complex vector periodic coefficients consisting of 31 elements (this corresponds to the number of Fourier terms taken in the expansion of 
L-F transformation matrix) with period $2 T$ corresponding to all possible monomials of order 3 in $y_{1}, y_{2}, y_{3} \& y_{4}$. Note that two of the eigenvalues in (60) are purely imaginary which is to be expected in this case. The centre manifold relations for this problem are assumed in the form

$$
\begin{aligned}
& y_{1}=B_{11}^{3}(t) y_{3}^{3}+B_{12}^{3}(t) y_{3}^{2} y_{4}+B_{13}^{3}(t) y_{3} y_{4}^{2}+B_{14}^{3}(t) y_{4}^{3}, \\
& y_{2}=B_{21}^{3}(t) y_{3}^{3}+B_{22}^{3}(t) y_{3}^{2} y_{4}+B_{23}^{3}(t) y_{3} y_{4}^{2}+B_{24}^{3}(t) y_{4}^{3}
\end{aligned}
$$

such that $B_{i j}^{3}(t), i=1,2 \& j=1,2,3,4$ are unknown periodic coefficients with period $2 T$. Note that in the above equation the states corresponding to stable eigenvalues are expressed in terms of the states corresponding to the critical eigenvalues.

Substituting (61) in (60), eight ordinary differential equations in $B_{i j}^{3}(t), i=1,2 \&$ $j=1,2,3,4$, similar to (40) are obtained. The periodic coefficients appearing on the right hand side of these differential equations are nothing but the known periodic coefficients corresponding to the cubic nonlinear terms appearing in (60). The unknown periodic coefficients $B_{i j}^{3}(t), i=1,2 \& j=1,2,3,4$ can be obtained by formally solving these differential equations. In order to obtain a particular solution, $B_{i j}^{3}(t)$ is assumed in the form of (41) with unknown constant coefficients and like terms on both sides of the equations are equated to obtain a set of linear algebraic equations in terms of the unknowns $a_{n}$ and $b_{n}$. The computation of all the unknowns of the $B_{i j}^{3}(t)$ 's requires the solution of a set of $8 \times 31$ linear algebraic equations. These algebraic equations can be solved such that each of the $B_{i j}^{3}(t), i=1,2 \& j=1,2,3,4$ can be obtained as Fourier series expansions. Noting that the problem under consideration consists of only cubic nonlinearities, it is not necessary to solve for all the periodic coefficients $B_{i j}^{3}(t), i=1,2 \& j=1,2,3,4$ in the centre manifold relation. Instead, it suffices to compute only one coefficient per relation in (61). This simplification does not affect the final outcome of the result, since the centre manifold relations result in nonlinearities which are of powers greater than three and does not influence the stability characteristics. Therefore, for this case, only coefficients $B_{11}^{3}(t)$ and $B_{21}^{3}(t)$ are computed.

Substitution of centre manifold relations (61) in (60) results in differential equations for the critical states $y_{3}$ and $y_{4}$ which contain nonlinearities of cubic and higher orders. Since the higher order terms do not affect the stability characteristics, the terms of order higher than 3 are neglected. The equations thus obtained are similar to (46) representing a Hopf bifurcation behaviour in a two-dimensional system with cubic nonlinearity. Following the procedure outlined earlier in the study of Hopf bifurcation of a single degree of freedom system, the application of time-dependent normal forms to these equations provide a simplified nonlinear equation similar to (53). The behaviour of the fixed point of the resulting equation is found to be a centre by employing similar methods to those outlined by Pandiyan (1994). For brevity, the calculations are not reported here. On the basis of the arguments presented for example 1, the motion resulting from the Hopf bifurcation is quasi-periodic and bounded. It can readily be seen that the Poincare plots provided in figures $5 \mathrm{a}$ and $5 \mathrm{~b}$ also confirm this result.

(ii) Flip bifurcation - Consider the parameters $\bar{k}=2.0, B_{1}=B_{2}=0.0175, P_{1}=0$, $P_{2}=0.5, \gamma=0.895, \omega=1.1$ such that one of the Floquet multipliers of (59) becomes 


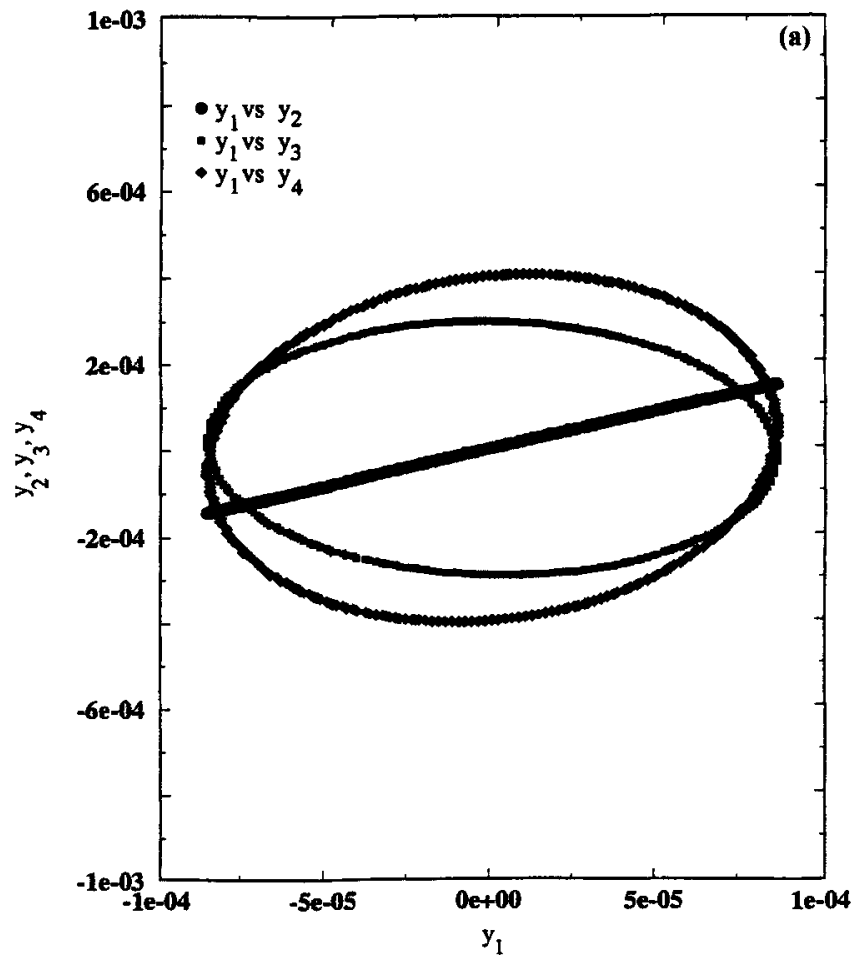

Figure 5. (a) Poincaré plots - Double inverted pendulum under Hopf bifurcation.

$-0.999938(\approx-1)$ and the system undergoes a flip bifurcation. After the transformation, the following Jordan canonical form is obtained

$$
\begin{aligned}
\left\{\begin{array}{l}
\dot{y}_{1} \\
\dot{y}_{2} \\
\dot{y}_{3} \\
\dot{y}_{4}
\end{array}\right\} & =\left[\begin{array}{cccc}
-0.1685+0.9557 i & 0 & 0 & 0 \\
0 & -0.1685-0.9557 i & 0 & 0 \\
0 & 0 & 0 & 0 \\
0 & 0 & 0 & -0.0137
\end{array}\right] \\
& \times\left\{\begin{array}{l}
y_{1} \\
y_{2} \\
y_{3} \\
y_{4}
\end{array}\right\}+\left\{\begin{array}{l}
\sum \mathbf{a}_{q}(t) y_{1}^{m_{1}} y_{2}^{m_{2}} y_{3}^{m_{3}} y_{4}^{m_{4}} \\
\sum \mathbf{b}_{q}(t) y_{1}^{m_{1}} y_{2}^{m_{2}} y_{3}^{m_{3}} y_{4}^{m_{4}} \\
\sum \mathbf{c}_{q}(t) y_{1}^{m_{1}} y_{2}^{m_{2}} y_{3}^{m_{3}} y_{4}^{m_{4}} \\
\sum \mathbf{d}_{q}(t) y_{1}^{m_{1}} y_{2}^{m_{2}} y_{3}^{m_{3}} y_{4}^{m_{4}}
\end{array}\right\}, \quad \sum_{i=1}^{4} m_{i}=3
\end{aligned}
$$

where $\mathbf{a}_{q}(t), \mathbf{b}_{q}(t), \mathbf{c}_{q}(t)$ and $\mathbf{d}_{q}(t)$ once again are vector periodic coefficients with period $2 T$. It is observed that the eigenvalue corresponding to the third state is zero and the remaining eigenvalues have negative real parts. The centre manifold relations for this case can be assumed in the form

$$
y_{1}=\mathbf{B}_{11}^{3}(t) y_{3}^{3} ; \quad y_{2}=\mathbf{B}_{21}^{3}(t) y_{3}^{3} ; \quad y_{4}=\mathbf{B}_{31}^{3}(t) y_{3}^{3},
$$

where $\mathbf{B}_{11}^{3}(t), \mathbf{B}_{21}^{3}(t)$ and $\mathbf{B}_{31}^{3}(t)$ are unknown coefficients with period $2 T$. These can be determined by solving the differential equations in $\mathbf{B}_{11}^{3}(t), \mathbf{B}_{21}^{3}(t)$ and $\mathbf{B}_{31}^{3}(t)$ as described 


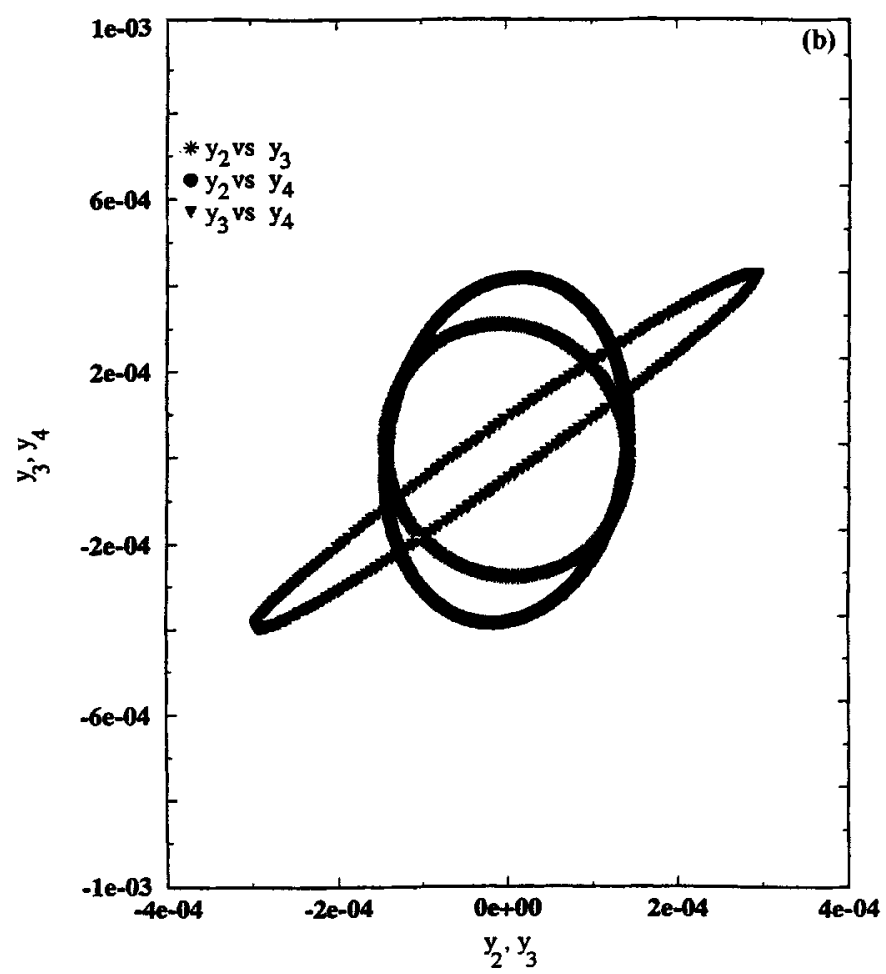

Figure 5. (b) Poincaré plots - Double inverted pendulum under Hopf bifurcation.

earlier. The computed coefficients are recorded by Pandiyan (1994). Substituting (63) into equation (62) and neglecting the higher order terms beyond the cubics, the one-dimensional centre manifold equation is found and since the mean value of the periodic coefficient of the reduced centre manifold equation for this case is positive, the fixed point is unstable and hence the corresponding $2 T$ periodic orbits in the original coordinates are unstable. In a similar way, the fold bifurcation of this system can also be studied. However, the results are not included here.

Financial support provided by the Army Research Office under the contract number DAAL03-94G-0337 is gratefully appreciated. The author would also like to acknowledge Dr R Pandiyan for his help in preparing the manuscript.

\section{References}

Arnold V I 1988 Geometrical methods in the theory of ordinary differential equations (New York: Springer-Verlag)

Awreicewicz J 1989 Bifurcation and chaos in simple dynamical systems (Singapore: World Scientific)

Bernussou J 1977 Point mapping stability (New York: Pergamon) 
Birkhoff G D 1966 Dynamical system (Providence: Am. Math. Soc.) vol. 9

Bogoliubov N N, Mitropolsky Yu A 1961 Asymptotic methods in the theory of nonlinear oscillations (New York: Gordon and Breach)

Bramwell A R S 1976 Helicopter dynamics (London: Edward Arnold)

Bruno A D 1989 Local methods in nonlinear differential equations (Berlin, Heidelberg: Providence: Springer-Verlag)

Carr J 1981 Applications of center manifold theory (New York: Springer-Verlag)

Chow S N, Wang D 1985 Normal forms of bifurcating periodic orbits. Contemporary Mathematics. Vol. 56 (Proceedings of a summer research conference, July 1985) (eds) M Golubitsky, J Guckenheimer (Providence, RI: Am. Math. Soc.)

Coddington E A, Levinson N 1955 Theory of ordinary differential equations (New York: McGraw Hill)

Cullum J, Willoughby R A (eds) 1986 A practical procedure for computing eigenvalues of large sparse nonsymmetric matrices. Large scale eigenvalue problem (New York: Elsevier Science)

Doedel E J, Kernévez J P 1986 AUTO: Software for continuation and bifurcation problems in ordinary differential equations. Applied Mathematics Report, California Institute of Technology

Flashner H, Hsu C S 1983 A study of nonlinear periodic systems via the point mapping method. Int. J. Numer. Meth. Eng. 19: 185-215

Floquét G 1883 Sur les équations differentials linéaires a coefficients périodiques. Annales Scientifiques de l'Ecole Normale Superieue series 2, vol. 12

Friedmann P, Hammond C E, Woo T H 1977 Efficient numerical treatment of periodic systems with application to stability problems. Int. J. Num. Meth. Eng. 11: 1117-1136

Gaonkar G H, Peters D A 1986 Review of Floquet theory in stability and response analysis of dynamic systems with periodic coefficients. Recent trend in aeroelasticity, structures, and structural dynamics (ed) P Hajela (Gainsville, FL: University of Florida Press) p 101

Gaonkar G H, Simha Prasad D S, Sastry D 1981 On computing Floquet transition matrices of rotorcraft. J. Am. Helicopter Soc. 26: 56-61

Guttalu R S, Flashner H 1989 Periodic solutions of nonlinear autonomous systems by approximate point mappings. J. Sound Vibr. 129: 291-311

Guttalu R S, Flashner H 1990 Analysis of dynamical systems by truncated point mapping and cell mapping. Nonlinear dynamics in engineering systems (ed) W Schiehlen (New York: SpringerVerlag)

Guckenheimer J, Holmes P 1983 Nonlinear oscillations, dynamical systems, and bifurcations of vector fields (New York: Springer-Verlag)

Hale J, Kocak H 1991 Dynamics and bifurcations (New York: Springer-Verlag)

Hsu C S 1987 Cell-to-cell Mapping (New York: Springer-Verlag)

Hsu C S, Cheng W H 1973 Applications of the theory of impulsive parametric excitation and new treatments of general parametric excitation problems. J. Appl. Mech. 40: 78-86

Hsu C S, Cheng W H 1974 Steady-state response of a dynamical system under combined parametric and forcing excitations. J. Appl. Mech. 41: 371-378

Jin J-D, Matsuzaki Y 1988 Bifurcations in a two-degree-of-freedom elastic system with follower forces. J. Sound and Vibr. 126: 265-277

Johnson W 1980 Helicopter theory (Princeton, NJ: University Press)

Joseph P, Pandiyan R, Sinha S C 1993 Optimal control of mechanical systems subjected to periodic loading via Chebyshev polynomials. Optimal control applications and methods $\mathrm{pp} 75-90$

Kretz M 1976 Research in multicyclic and active control of rotary wings. Vertica 1: 95-105

Lalanne M, Ferraris G 1990 Rotordynamics prediction in engineering (Chichester: John Wiley and Sons) 
Lindh K G, Likins P W 1970 Infinite determinant methods for stability analysis of periodiccoefficient differential equations. AIAA J. 8: 680-686

Lindtner E, Steindl A, Troger H 1990 Generic one-parameter bifurcations in the motion of a simple robot. Continuation techniques and bifurcation problems (ISNM 92) (eds) H D Mittelmann, D Roose (Birkhauser); also in J. Comput. Appl. Math. (1989) 26: 199-218

Lukes D L 1982 Differential equations: Classical to controlled (New York: Academic Press)

Malkin I G 1962 Some basic theorems of the theory of stability of motion in critical cases. Stability and dynamic systems, Translations, American Mathematical Society, Series 1 (Am. Math. Soc.) 5: 242-290

Mckillip R M Jr 1985 Periodic control of individual-blade-control helicopter rotor. Vertica 9: 199-225

Nayfeh A H 1973 Perturbation methods (New York: Wiley)

Pandiyan R 1994 Analysis and control of nonlinear dynamic systems with periodically varying parameters. $\mathrm{Ph} \mathrm{D}$ dissertation, Dept. Mechanical Engineering, Auburn University

Pandiyan R, Sinha S C 1995 Analysis of time-periodic nonlinear dynamical systems undergoing bifurcations. Nonlinear Dynamics 8: 21-43

Pandiyan R, Bibb J S, Sinha S C 1993 Liapunov-Floquet transformation: Computation and application to periodic systems. In Dynamics and vibration of time-varying systems and structures 14th ASME Biennial Conference on Mechanical Vibration and Noise (eds) S C Sinha, R M Evan-Iwanowski (New York: ASME Press) pp 337-348; also in J. Vibr. Acoust. 118: 209-219

Peters D A, Hohenemser K H 1971 Application of the Floquet transition matrix to problems of lifting rotor stability. J. Am. Helicopter Soc. 16: 25-33

Poincaré H 1899 Les Methodes Nouvelles de la Mécanique Céleste (Paris: Gauthier-Villars)

Rosenblat S, Cohen D S 1980 Periodically perturbed bifurcation I. Simple bifurcation. Studies in Appl. Math. 63: 1-23

Rosenblat S, Cohen D S 1981 Periodically perturbed bifurcation II. Hopf Bifurcation. Studies in Appl. Math. 65: 95-112

Sanders J A, Verhulst F 1985 Averaging methods in nonlinear dynamical systems (New York: Springer-Verlag)

Sethna P R, Schapiro S M 1977 Nonlinear behavior of flutter unstable dynamical systems with gyroscopic and circulatory forces. J. Appl. Mech. 44: 755-762

Seydel R 1981 Numerical computation of periodic orbits that bifurcate from stationary solutions of ordinary differential equation. Appl. Math. Comput. 9: 257-271

Seydel R 1987 New methods for calculating stability of periodic solutions. Comput. Math. Appl. 14: 505-510

Seydel R 1988 From equilibrium to chaos: Practical bifurcation and stability analysis (New York: Elsevier)

Sinha S C, Joseph P 1994 Control of general dynamic systems with periodically varying parameters via Liapunov-Floquet Transformation. ASME J. Dynamic Syst., Measurements Control 116: 650-658

Sinha S C, Juneja V 1991 An approximate analytical solution for systems with periodic coefficients via symbolic computation. AIAA/ASME/ASCE/AHS/ASC 32nd Structures, Structural Dynamics and Materials Conference (A Collection of Papers, Part 1) pp 790-797

Sinha S C, Pandiyan R 1994 Analysis of quasilinear dynamical systems with periodic coefficients via Liapunov-Floquet transformation. Int. J. Non-Linear Mech. 29: 687-702

Sinha S C, Wu D-H 1991 An efficient computational scheme for the analysis of periodic systems. J. Sound Vibr. 15: 345-375 
Sinha S C, Wu D-H, Juneja V, Joseph P 1993a Analysis of dynamic systems with periodically varying parameters via Chebyshev polynomials. ASME J. Vibr. Acoust. 115: 96-102

Sinha S C, Senthilnathan N R, Pandiyan R 1993b A new numerical technique for the analysis of parametrically excited nonlinear systems. Nonlinear Dynamics 4: 483-498

Stoker J J 1950 Nonlinear vibration (New York: Interscience)

Wu D-H, Sinha S C 1994 A new approach in the analysis of linear systems with periodic coefficients for applications in rotorcraft dynamics. Aeronaut. J. R. Aeronaut. Soc. January: 9-16

Yakubovitch V A, Starzhinskii V M 1975 Linear differential equations with periodic coefficients (New York: Wiley-Halsted) vols. 1 and 2 